\title{
Gene genealogy in a population of variable size
}

\author{
BRUCE RANNALA \\ Department of Integrative Biology, University of California, Berkeley, CA 94720-3140, U.S.A.
}

\begin{abstract}
The genealogical properties of a small population with continuous overlapping generations that fluctuates randomly in size are studied using a model based on a stochastic birth-death process. The distribution of the coalescence times is presented, as well as a method for computing the expected overall length of the genealogy as a function of the individual birth rate $\lambda$, the individual death rate $\mu$, and the present population size. The relationship between the birth and death rates and the shape of the resulting genealogy is studied. The total length of the genealogy is shown to be maximized when $\lambda=\mu$. The joint distribution of the coalescence times is shown to be invariant in $\lambda$ and $\mu$, conditional on the current population size, so that exponential growth of a population cannot be distinguished from exponential decline based on the shape of the resulting genealogy. The model is used to predict the probability that all genetic variation is lost from a recent founder population.
\end{abstract}

Keywords: birth-death process, conservation genetics, founder population, gene coalescent, genetic diversity, stochastic demography.

\section{Introduction}

Changes in population size, whether random or systematic, may have an important influence on genetic structure and effective population size. In growing or declining populations, this is reflected by changes in the shape of allelic genealogies under a coalescent process (Kingman, 1982) which traces the ancestry of alleles. Recently, a number of authors have studied the genealogical properties of a Wright-Fisher model with deterministic size variation (Slatkin \& Hudson, 1991; Rogers \& Harpending, 1992; Griffiths \& Tavaré, 1994; Nee et al., 1995). Slatkin \& Hudson (1991) showed that genealogies in populations expanding at an exponential rate have coalescence times concentrated over a restricted range of the genealogical tree (i.e. are more 'starlike'). Griffiths \& Tavaré (1994) derived the joint distribution of the times between coalescence events in a population undergoing deterministic expansion.

In this paper, I consider the genealogical properties of a stochastic demographic model for a population closed to immigration and with a variable size determined by a linear birth and death process. Karlin \& McGregor (1967), Kendall (1975), Tavaré

*Correspondence. E-mail: bruce@mws4.biol.berkeley.edu
(1989) and Rannala (1996) have previously studied the distribution of marked types (i.e. marking as a result of either mutation of immigration) for the linear birth, death and immigration process, originally proposed by Kendall (1948) as a model of populations open to immigration and of variable size. The genetic properties of demographic models of supercritical branching processes, which are discretetime analogues of birth-death (BD) processes (with $\lambda>\mu$ ), have also been studied (Taïb, 1992).

The linear birth and death model of population demography is similar to a model proposed by Moran (1958) to study genetic structure in populations with overlapping generations. The Moran model considers a Markov process in which each event, in discrete time, is a birth whose parent is chosen at random from the population, followed immediately by a random death. The total population size under the Moran model remains constant over time. The BD process allows for random deaths as well as births; birth and death events occur, for any particular individual, according to independent Poisson processes and the total population size fluctuates over time.

The linear BD process provides a reasonable model for studying populations in which individual birth and death rates are independent of population density. This might be expected to be the case in 
populations well below the environmental carrying capacity; examples could include founder populations that have invaded new habitats, and relict populations that are declining because of human exploitation. The $\mathrm{BD}$ process is also potentially useful for describing the population dynamics of a newly mutated allele at low frequency in a population.

For large $N$, if $\lambda \neq \mu$, the change in population size over time under a BD process is adequately described using a deterministic model of exponential population growth $(\lambda>\mu)$ or decline $(\lambda<\mu)$. The well-known result for the population size at time $t$ under the deterministic process is equal to the expectation under the stochastic BD model (see below):

$$
\mathrm{E}[N(t)]=N(0) \mathrm{e}^{(i-\mu) t},
$$

where $N(0)$ is the population size at time $t=0$. Griffiths \& Tavaré (1994) provide a general result for the distribution of coalescence times in a population with nonoverlapping generations and a population size that changes as a deterministic function of time. The results of Griffiths \& Tavaré are based on a diffusion approximation; for large populations the properties of a birth-death process should be well described using their results (with an appropriate change of time-scale). In many cases, however, it is important to consider specifically the genealogical properties of small populations for which a diffusion approximation may not be valid.

In general, populations in nature that satisfy the density-independent growth assumption of the BD model will be quite small in size (human populations are an obvious exception). In particular, many species of interest to conservation biologists may be declining in size because of exploitation at rates roughly proportional to the total population numbers. For such populations, a demographic model that takes into account stochastic size fluctuations in modelling the genetic structure may be expected to be more accurate than a deterministic model. For moderately small values of $N, \lambda$ and $\mu$, the population coalescence times (measured in units of expected births) are recent enough that mutation will be of negligible importance as a force determining genetic variation. In this study, I focus primarily on the probability that pre-existing variation in a population is lost under a BD process. The results presented for the gene genealogy are also relevant to larger populations and genetic models that incorporate mutation, however, and allow some predictions concerning the shapes of genealogies reconstructed using molecular data.

\section{Theory}

\section{Demographic model}

The linear BD process was first described by Feller (1939), and many of its mathematical properties were studied by Kendall (1949). The process may be formulated as follows: during an infinitesimal time interval $\delta t$, the probability that a birth occurs in a population of size $N$ is $N \lambda \delta t$ and the probability that a death occurs is $N \mu \delta t$. The probability of two or more events is of order $\mathrm{O}(\delta t)$ and may be neglected. Note that $\lambda$ is the individual birth rate and $\mu$ is the individual death rate. There is a positive probability of population extinction under this process for finite (and nonzero) values of $\lambda$ and $\mu$. Because $\mathrm{I}$ am primarily interested in the genealogy arising under the model, I consider only cases in which the population has not become extinct.

If a population is initiated by a single individual (i.e. $N(0)=1)$ the probability that the population is extinct at time $t>0$ is (from Kendall, 1949)

$p_{0}(t)=\frac{\mu\left[e^{(\hat{\lambda}-\mu) t}-1\right]}{\lambda e^{(\lambda-\mu) t}-\mu}$.

The probability that the population consists of $N$ descendents at time $t$ is

$p_{N}(t)=\left[1-p_{0}(t)\right]\left(1-\eta_{t}\right) \eta_{t}^{N-1}$,

where $\eta_{t}=p_{0}(t)(\lambda / \mu)$. The expectation and variance of $N(t)$ are

$\mathrm{E}[N(t)]=e^{(\hat{\lambda}-\mu) t}$,

$\operatorname{Var}[N(t)]=\frac{\lambda+\mu}{\lambda-\mu} e^{(\lambda-\mu) t}\left(e^{(\hat{\lambda}-\mu) t}-1\right)$.

If $N(0)>1$, each individual has an independently distributed number of offspring (with expectation and variance as given in eqns 4 and 5) and we obtain the result for the expected population size presented in eqn (1).

\section{Gene genealogy}

In this paper, I focus on the genealogy of a collection of $N \geq 2$ haploid individuals, observed at time present. Nee et al. (1994) refer to this as the genealogy of the 'reconstructed process' and it may be visualized as a tree of ancestors and descendents under a BD process with the lineages extinct before time present removed (Fig. 1). To be consistent with previous work, I will denote the time at present as zero and the time at which all the lineages coalesce 


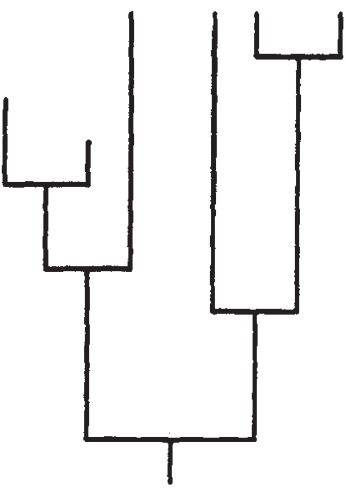

A

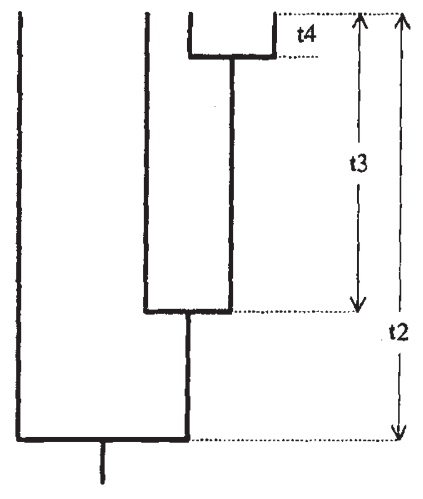

B
Fig. 1 Two possible representations of the genealogy of a $\mathrm{BD}$ process. The genealogy at left, denoted as $\mathrm{A}$, is complete and includes six individuals, only four of whom are alive at time $t=0$. The genealogy at right, denoted as $\mathrm{B}$, only includes the four individuals alive at time $t=0$. The waiting times $t_{4}, t_{3}$ and $t_{2}$ until the four survivors coalesce to three ancestors, two ancestors and one ancestor, respectively, are shown at right.

to a single ancestor as $t_{2}>0$. A population of $N$ lineages, under this process, coalesces to $N-1$ ancestral lineages at time $t_{N}, N-2$ lineages at time $t_{N-1}$ and so on. Coalescence events occur at $N-1$ distinct times in total (Fig. 1b). The first coalescence event occurs at time $t_{N}$ and the last at time $t_{2}$ when only a single ancestor remains. The joint distribution of coalescence times under the BD process was originally derived in a different context by Thompson (1975) and later by Nee et al. (1994). My derivation differs, however, in that I do not condition on the time of the final coalescence event.

\section{Distribution of coalescence times}

A population of $N$ individuals, alive at time present, is descended through $N-1$ coalescence events from a single ancestor. The probability that an individual is born to one of $i-1$ lineages existing at time $t_{i}$ that ultimately survive to time present, and the new lineage is represented by a single descendent lineage at present is

$(i-1) \lambda p_{1}\left(t_{i}\right)$,

where (from Kendall, 1949),

$p_{1}\left(t_{i}\right)=\left(1-p_{0}\left(t_{i}\right)\right)\left(1-(\lambda / \mu) p_{0}\left(t_{i}\right)\right)$.

This result follows as the $i$ individuals alive at time $t_{i}$ that survive to present are equally likely to give birth to the new lineage; the new lineage survives to leave a single descendent at time present with probability $p_{1}\left(t_{i}\right)$. The joint density of observing $N$ lineages at time present that arise at times $t_{N}<t_{N-1}<\ldots<t_{2}$ in the past and are descended from a single ancestral lineage that appeared at time $t_{1}$, conditional on the present population size $N$, is then

$$
\begin{aligned}
\mathrm{P}\left(t_{N}, \ldots, t_{2} \mid \lambda, \mu ; t_{1}, N\right) & =\frac{p_{1}\left(t_{1}\right)}{p_{N}\left(t_{1}\right)} \prod_{i=2}^{N}(i-1) \lambda p_{1}\left(t_{i}\right) \\
& =(N-1) ! \prod_{i=2}^{N}\left\{\mu \frac{p_{1}\left(t_{i}\right)}{p_{0}\left(t_{1}\right)}\right\} .
\end{aligned}
$$

An interesting property of eqn (8) is that the joint density of coalescence times is invariant in $\mu$ and $\lambda$. This result has been independently observed by $\mathrm{M}$. Slatkin (personal communication) who used an approach different from the one presented in this paper. This invariance can be shown by comparing the joint density of coalescence times for a growing population with parameters $\lambda_{1}>\mu_{1}$ and a declining population with parameters $\mu_{2}>\lambda_{2}$. The densities are identical if $\lambda_{1}=\mu_{2}$ and $\lambda_{2}=\mu_{1}$. This implies that the shape of the genealogy of alleles is identical for populations either growing, or declining, with identical but opposite rates; population growth can therefore not be distinguished from population decline using only the allelic genealogy. It is clear from eqn (8) that the joint density of coalescence times is equivalent to the joint density of the order statistics (i.e. $\left.x_{(2)}>x_{(3)}>\ldots>x_{(N)}\right)$ of $N-1$ independent and identically distributed (iid) random variables with density

$f_{x}(x)=\frac{\mu p_{1}(x)}{p_{0}\left(t_{1}\right)}$.

The marginal or joint densities of the $t_{2}, \ldots, t_{N}$ coalescence times are identical to those of the order statistics of the variables $x$ and various properties of these may be studied using standard methods. In particular, the marginal density of the $j$ th coalescence time is given by

$\mathrm{P}\left(t_{j} \mid \lambda, \mu\right)=\frac{(N-1) !}{(N-j) !(j-2) !} f_{x}\left(t_{j}\right)\left[F_{x}\left(t_{j}\right)\right]^{N-j}\left[1-F_{x}\left(t_{\mathrm{j}}\right)\right]^{j-2}$,

where $F_{x}(x)$ is the cumulative distribution function (c.d.f) of the random variable $x$ given by

$$
\begin{aligned}
F_{x}(x) & =\int_{0}^{x} f_{x}(y) d y \\
& =\frac{\left(e^{x(\hat{\lambda}-\mu)}-1\right)\left(\mu-\lambda e^{t_{1}(\hat{\lambda}-\mu)}\right)}{\left(e^{t_{1}(\hat{\lambda}-\mu)}-1\right)\left(\mu-\lambda e^{x(\hat{i}-\mu)}\right)} .
\end{aligned}
$$


The marginal density of $t_{2}$ is of particular interest; this is the time at which all individuals coalesce to a single ancestor and all genetic variation is lost from the population (see discussion below).

If the ancestor of all individuals arose at a time $t_{1} \rightarrow \infty$ in the past, by taking the limit of eqn (8) we obtain

$\mathrm{P}\left(t_{N}, \ldots, t_{2} \mid \lambda, \mu, N\right)=\left\{\begin{array}{l}(N-1) ! \Pi_{i=2}^{N} \lambda p_{t}\left(t_{i}\right) \text { if } \lambda>\mu \\ (N-1) ! \Pi_{i=2}^{N} \mu p_{1}\left(t_{i}\right) \text { if } \mu>\lambda\end{array}\right.$

This is the limiting distribution of coalescence times in a population, currently of size $N$, whose ultimate ancestral lineage arose at a time far in the past. Using a logic similar to that applied for the case of finite $t_{1}$, the joint density of $t$, in the limit as $t_{1} \rightarrow \infty$, is given as the order statistics of a collection of $N-1$ iid random variables with density $f_{x}(x)=B p_{1}(x)$, where $B=\lambda$ if $\lambda>\mu$ and $B=\mu$ if $\mu>\lambda$. Marginal densities may then be calculated using eqn (10) above.

\section{Genealogy length}

The total length of the allelic genealogy (the sum over all branches) in a population provides a useful summary of genetic structure (Tavaré, 1984). If one considers the DNA sequence for a particular gene in the population, allowing for mutations arising according to the infinite sites model of nucleotide substitution (Kimura, 1969), then the expected number of segregating sites among individuals is a linear function of the genealogy length (Hudson, 1990). Over shorter time scales, for which mutational effects may be negligible, the genealogy length is inversely related to the probability that the variability among individuals for a particular gene is lost (see below). The total length of the genealogy, summing over all branches, may be derived as the following function of the coalescence times:

$T=\sum_{i=2}^{N} t_{i}+t_{2}$

For a BD process, we have the simple result that the expectation of $T$ is

$$
\begin{aligned}
\mathrm{E}[T] & =\sum_{i=2}^{N} \mathrm{E}\left[t_{i}\right]+\mathrm{E}\left[t_{2}\right] \\
& =(N-1) \mathrm{E}[x]+\mathrm{E}\left[t_{2}\right],
\end{aligned}
$$

where the expectation of $x$ is

$\mathrm{E}[x]=\int_{0}^{t_{1}} y f_{x}(y) d y$

$$
= \begin{cases}\frac{\lambda(\lambda-\mu) e^{t_{1}(i-\mu)} t_{1}+\left(e^{t_{1}(\lambda-\mu)} \lambda-\mu\right) \log \left\{\frac{\mu-\lambda}{\mu-\lambda e^{t_{1}(\lambda-\mu)}}\right\}}{\lambda \mu\left(1-e^{t_{1}(\bar{\lambda}-\mu)}\right)} & \text { if } \lambda \neq \mu \\ \frac{\left(1+\lambda t_{1}\right) \log \left(1+\lambda t_{1}\right)-\lambda t_{1}}{\lambda^{2} t_{1}} & \text { if } \lambda=\mu .\end{cases}
$$

The expected time of the final coalescence event is

$\mathrm{E}\left[t_{2}\right]=\int_{0}^{\infty} t_{2} \mathrm{P}\left(t_{2} \mid \lambda, \mu\right) d t_{2}$

where $\mathrm{P}\left(t_{2} \mid \lambda, \mu\right)$ is the marginal density calculated using eqs (9-11). The integral of eqn (16) does not appear to have an analytical solution, but may be evaluated numerically using standard methods. In the examples below, I use numerical integration routines in the Mathematica computer program (Wolfram Research, 1992). A Mathematica package for performing these calculations is available on the internet web page at http://mw511.biol.berkeley.edu/ homepage.html.

The effect of changing birth and death rates on the total genealogy length in a population of current size $N=50$ and with $t_{1}=10$ is shown in Fig. 2. The lower curve is for the case where the death rate is constant $(\mu=1)$ and the birth rate is increasing; this corresponds to an increase in the overall rate of growth for the population. The effect is an approximately exponential decrease in the total length of the genealogy with a linear increase in $\lambda$. These

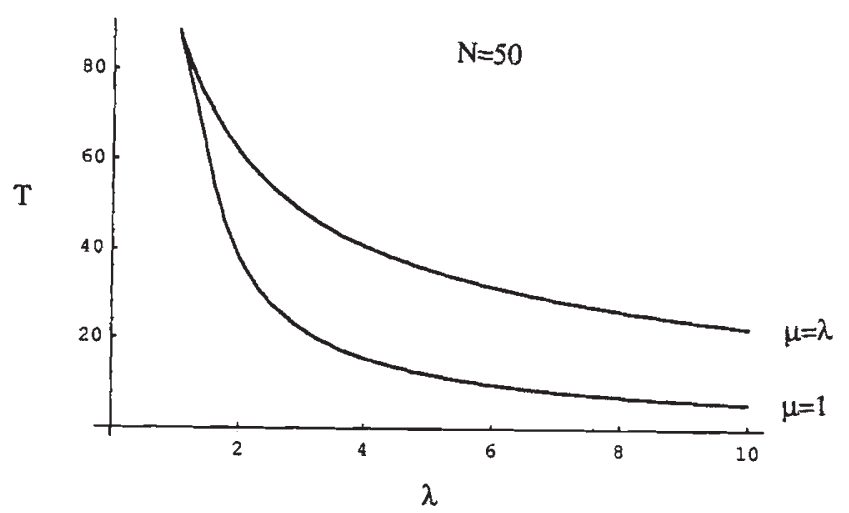

Fig. 2 Total length of the population genealogy $T$ as a function of the individual birth rate $\lambda$. The population size is $N=50$ in all cases and the age of the ultimate ancestral lineage is $t_{1}=10$. The upper curve is for the case where the individual birth rate $\lambda$ equals the death rate $\mu(\mu=\lambda)$; the lower curve is for the case $\mu=1$. 
results are similar to those observed by Griffiths \& Tavaré (1994) for a Wright-Fisher model with deterministic population growth. Their results predict that the expected coalescence times (on a time scale proportional to the current population size) decrease with an increase in the population growth rate.

The upper curve shows the expected genealogy length for the case where $\lambda=\mu$ and the magnitude of $\lambda$ is increased; this corresponds to an increase in the reproductive variance among individuals (and an increase in the variance of the total population size over time), although the expectation of the total population size over time remains constant. The effect of this increase in the reproductive variance is to decrease the total genealogy length, but with less influence than when $\mu$ is fixed. The total length of the genealogy for a population with $\lambda=\mu=c$ is always greater than for either $\lambda=c>\mu$ or $\mu=c>\lambda$. These results agree with classical theory on the effects of population size fluctuations and variance in reproductive success on population genetic variability.

\section{Demography and genealogy shape}

To evaluate the effect of a stochastic increase, or decrease, of total population size on the shape of the genealogy resulting under a $\mathrm{BD}$ process, I considered the influence of $\lambda$ and $\mu$ on the function $\operatorname{Var}\left(x \mid t_{1}=1\right)$. This function gives the variance among coalescence times $t_{2}, \ldots, t_{N}$ relative to $t_{1}$, the age of the initial ancestor. If this variance is smaller, the coalescence times are concentrated over a more restricted time range and the tree is more 'starlike'. In the extreme case, where $\operatorname{Var}\left(x \mid t_{1}=1\right)=0$, we have a star genealogy with all lineages arising at precisely the same time. For large $N$, the approximate interval containing 95 per cent of the coalescence events is $\sqrt{\operatorname{Var}\left(x \mid t_{1}=1\right)} \times 1.96$. The lower curve in Fig. 3 shows the change in $\operatorname{Var}\left(x \mid t_{1}=1\right)$ with increasing $\lambda$ and with $\mu=10$. The coalescence events become concentrated over a smaller interval of time relative to $t_{1}$ (i.e. $\operatorname{Var}\left(x \mid T_{1}=1\right)$ decreases) in a population with increasing $\lambda$ (or equivalently decreasing $\mu$ ). The general effect of increasing $\lambda$ is then to produce a more starlike genealogy as predicted by Slatkin \& Hudson (1991) for the genealogy shape expected under a Wright-Fisher model with deterministic population growth.

The upper curve in Fig. 3 shows the change in $\operatorname{Var}\left(x \mid t_{1}=1\right.$ ) with increasing $\lambda$ (or equivalently $\mu$ ), but holding the difference between the parameters $\lambda$ and $\mu$ constant (i.e. in the example of Fig. 3:

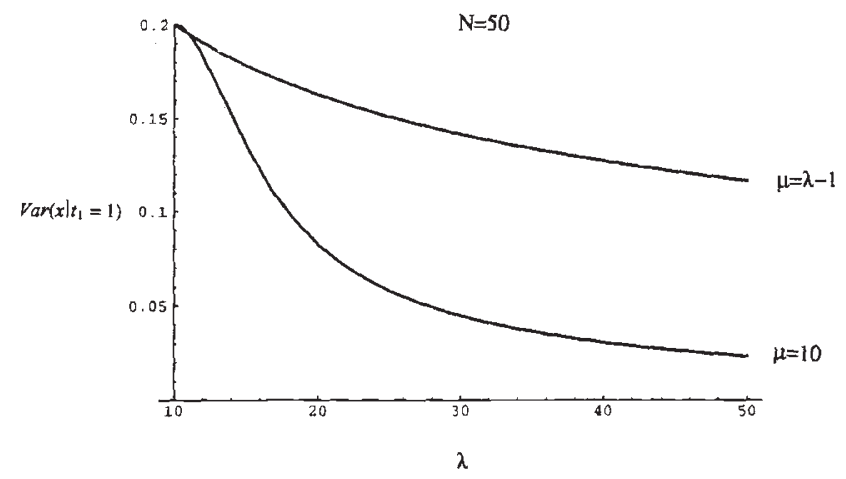

Fig. 3 Variance of coalescence times relative to $t_{1}$, the time of origin of the ultimate ancestral lineage $\left(\operatorname{Var}\left(x \mid t_{1}=1\right)\right)$, as a function of the individual birth rate $\lambda$. The population size is $N=50$ in all cases. The upper curve is for the case where the difference between the birth rate and the death rate is a constant $(\mu=\hat{\lambda}-1)$; the lower curve is for the case $\mu=10$.

$\lambda-\mu=1)$. In this case, the average rate of population growth remains constant, but the variance of the population size over time increases (as well as the variance in reproductive success among individuals). This case also results in a reduction of $\operatorname{Var}\left(x \mid t_{1}=1\right)$ (i.e. an increased concentration of coalescence times). This finding is different from the expectation based on a well-known result for the Wright-Fisher coalescent that shows an increase in reproductive variance among individuals $\sigma^{2}$ is equivalent to a fixed reduction in population size (i.e. the time scaling of the overall process is altered by a factor $1 / \sigma^{2}$ ) but does not otherwise affect the genealogy shape (Kingman, 1982). For a BD process, an increase in the reproductive variance among individuals also results in an increase in the magnitude of population size fluctuations over time; this appears to affect the genealogy shape as well as the effective population size, resulting in a genealogy that is more starlike.

\section{Genetic diversity in a founder population}

Consider a population that was founded by an unknown number of individuals at time $t_{\mathrm{f}}$ in the past. It is assumed that we are able to estimate the present population size, the birth rate $\lambda$, and the death rate $\mu$, using ecological field studies, and that individual age is not an important factor influencing reproduction and survival. An important question from the perspective of conservation genetics is whether all of the original genetic variability among the population founders has been lost by the present time. Clearly if all the lineages coalesce before time 
$t_{\mathrm{f}}$, then no genetic variability will remain in the population. This probability is given by

$\mathrm{P}\left(t_{2} \leqslant t_{\mathrm{f}}\right)=F_{t_{2}}\left(t_{\mathrm{f}}\right)$,

where $F_{t_{2}}$ is the c.d.f. of $t_{2}$ derived using eqs (10 and $12)$. The probability that all diversity is retained in the population is equal to the probability that the first coalescence event occurs after $t_{\mathrm{f}}$ (i.e. $t_{N}>t_{\mathrm{f}}$ ). This probability is given by

$$
\begin{aligned}
\mathrm{P}\left(t_{N}>t_{\mathrm{f}}\right) & =1-\mathrm{P}\left(t_{N} \leqslant t_{\mathrm{f}}\right) \\
& =1-F_{t_{N}}\left(t_{\mathrm{f}}\right) .
\end{aligned}
$$

The probability that all diversity is lost, as a function of $\lambda$, is shown for a growing population $(\lambda>\mu)$ in Fig. 4 for a range of values of $\mu$ and $N$. The probability that all original diversity is retained $\left(1-F_{t_{N}}\left(t_{f}\right)\right)$ is essentially zero, over most reasonable ranges of the parameters, suggesting that this is a less informative measure of genetic structure; the probability that at least one coalescence event occurs is virtually certain in most cases. In general, it appears that the probability of a complete loss of diversity (i.e. that the populations coalesce to a single founder lineage) increases with decreasing population size, increasing birth rate or the time elapsed since the founder event. This may be explained by noting that the results are conditional on the present population size; if the population size is fixed and the birth rate is increased, this increases the probability that few founders were present at time $t_{\mathrm{f}}$. Similarly, decreasing the current population size and keeping $\lambda$ constant again increases the probability of few founders.

\section{Discussion}

The results of this study suggest that a number of the general findings of earlier studies that have considered the genealogical structure of large populations with deterministic variation in total population size will also be true for small, stochastically fluctuating populations. In particular, the genealogy shape becomes more starlike with an increase in the birth rate relative to the death rate (Slatkin \& Hudson, 1991; Rogers \& Harpending, 1992). However, a new finding of this study is that genealogies in populations that are either growing, or declining, with similar rates are more starlike than genealogies for populations with a constant expected size. This suggests that genealogy shape alone is not sufficient to detect a rapid population growth. It should be noted, however, that this invariance of the genealogy shape for growing, or declining, populations is conditional on the current population size. The current population size may be much more probable for $\lambda>\mu$ or vice versa; the joint density of coalescence times and population size $N$ is not invariant in $\lambda$ and $\mu$.

Two possible predictors of the genetic variability in an expanding founder population, or a declining relict population, were also considered in this study.
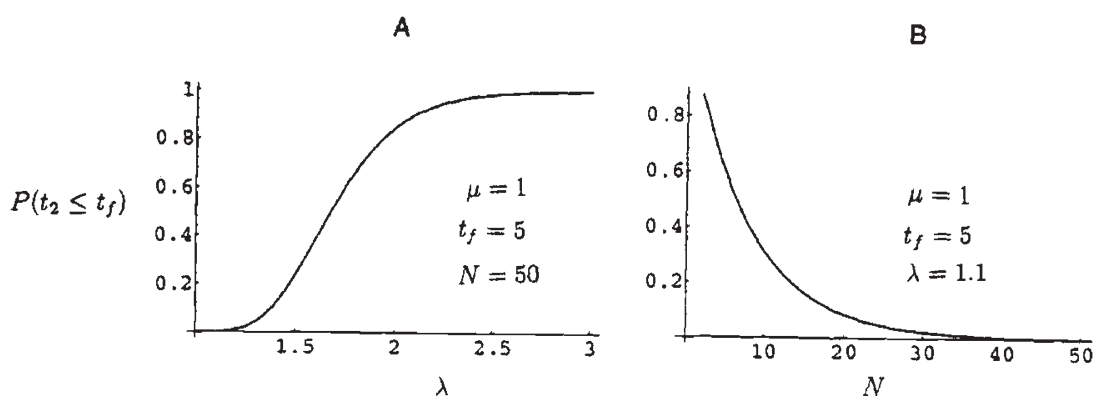

c

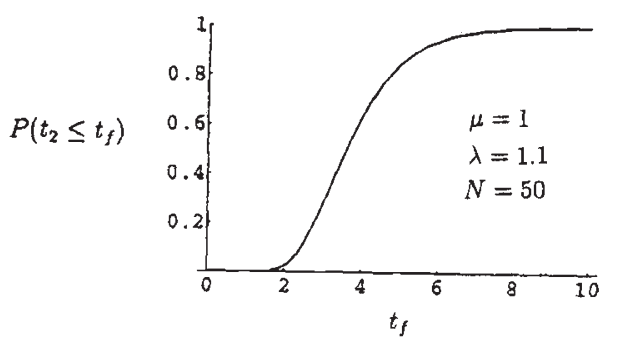

$D$

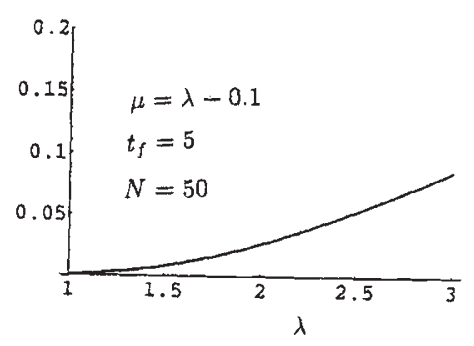

Fig. 4 Probability that all diversity is lost from a founder population $\left(P\left(t_{2} \leqslant t_{\mathrm{f}}\right)\right)$. Graph A at the upper left shows the effect of an increase in the individual birth rate $\lambda$ on the probability of a loss of diversity $P\left(t_{2} \leqslant t_{\mathrm{f}}\right)$, with the mutation rate $\mu=1$, the time of population founding at $t_{\mathrm{f}}=5$ and the population size at $N=50$. Graph $B$ at the upper right shows the effect of an increase in population size $N$ on $P\left(t_{2} \leqslant t_{\mathrm{f}}\right)$, with $\mu=1, t_{\mathrm{f}}=5$ and $\lambda=1.1$. Graph $\mathrm{C}$ at the lower left shows the effect of the time of the population founding event $t_{\mathrm{f}}$ on $P\left(t_{2} \leqslant t_{\mathrm{f}}\right)$, with $\mu=1, \lambda=1.1$ and $N=50$. Graph D at the lower right shows the effect of an increase in the individual birth rate $\lambda$ on $P\left(t_{2} \leqslant t_{\mathrm{f}}\right)$ when the difference between the birth rate and the death rate $\mu$ is held constant at $\mu=\lambda-0.1$, where $t_{\mathrm{f}}=5$ and $N=50$. 
The c.d.f. of the final coalescence time gives the probability that all genetic variability has been lost from the population, whereas the c.d.f. of the first coalescence event gives the probability that all original genetic diversity has been retained. The probability of a complete loss of diversity increases with a decrease in the total population size, an increase in the individual birth rate, or an increase in the total time elapsed since the founding event occurred. The probability that all diversity among founders is preserved is effectively zero over the range of parameters studied, suggesting that this measure is less informative.

The BD process appears to provide a useful model for studying the genetic structure arising in small populations with overlapping generations that are highly variable in size, are not strongly agestructured, and are not regulated by density-dependent effects. The model should prove particularly helpful in studying many problems arising in conservation genetics where a large population size assumption may not be warranted and stochastic effects arising from population size variation are important. The probability distribution of coalescence times for the $\mathrm{BD}$ process considered in this study might also be applied to estimate the birth and death rates in a population using DNA sequences for one or more genes. The calculation would involve integrating and summing over large numbers of variables and would be similar to the method implemented by Kuhner et al. (1995) to estimate jointly the effective population size and mutation rate under a Wright-Fisher coalescent model. Such calculations for a BD process do not appear feasible at the present time.

\section{Acknowledgements}

Support was provided by a Natural Sciences and Engineering Research Council (NSERC) of Canada postdoctoral fellowship, and in part by a grant from the National Institutes of Health (GM-40282) to M. Slatkin. I thank S. Otto, M. Slatkin and an anonymous reviewer for their helpful comments on an earlier version of this paper.

\section{References}

FELl_ER, w. 1939. Die grundlagen der volterraschen theorie des kampfes ums dasein in wahrsheinlichkeits theoretischen behandlung. Acta Biotheoret., 5, 1-40.

GRIfFITHS, R. C. AND TAVARÉ, S. 1994. Sampling theory for neutral alleles in a varying environment. Phil. Trans. $R$. Soc. B, 344, 403-410.

HUDSON, R. R. 1990. Gene genealogies and the coalescent process. Oxford Surveys Evol. Biol., 7, 1-44.

KARLIN, S. AND MCGREGOR, J. 1967. The number of mutant forms maintained in a population. 5th Berkeley Symposium on Mathematical Statistics and Probability, pp. 415-438. University of California Press, Berkeley, CA.

KENDALL, D. G. 1948. On some modes of population growth leading to R. A. Fisher's logarithmic series distribution. Biometrika, 35, 6-15.

KENDALl., D. G. 1949. Stochastic processes and population growth. J. R. Statist. Soc. B, 11, 230-264.

KENDALI, D. G. 1975. Some problems in mathematical genealogy. In: Gani, J. (ed.) Perspectives in Probability and Statistics: Papers in Honour of M. S. Bartlett on the Occasion of his Sixty-Fifth Birthday, pp. 325-348. Applied Probability Trust, New York.

KIMURA, M. 1969. The number of heterozygous nucleotide sites maintained in a finite population due to steady flux of mutations. Genetics, 61, 893-903.

KINGMAN, J. C. 1982 . On the genealogy of large populations. J. Appl. Prob., 19A, 27-43.

KUHNER, M. K., YAMATO, J. AND FELSENSTEIN, J. 1995. Estimating effective population size and mutation rate from sequence data using Metropolis-Hastings sampling. Genetics, 140, 1421-1430.

MORAN, P. A. P. 1958. The general theory of the distribution of gene frequencies. I. Overlapping generations. II. Non-overlapping generations. Proc. R. Soc. B, 149, 102-116.

NEE, S., HOLMES, E. C., RAMBAUT, A. AND HARVEY, P. H. 1995. Inferring population history from molecular phylogenies. Phil. Trans. R. Soc. B, 349, 25-31.

NEE, S., MAY, R. M. AND HARVEY, P. H. 1994. The reconstructed evolutionary process. Phil. Trans. R. Soc. B, 344, 305-311.

RANNALA, B. 1996. The sampling theory of neutral alleles in an island population of fluctuating size. Theoret. Pop. Biol., 50, 91-104.

ROGERS, A. R. AND HARPENDING, H. 1992. Population growth makes waves in the distribution of pairwise genetic differences. Mol. Biol. Evol., 9, 552-569.

SLATKIN, M. AND HUDSON, R. R. 1991. Pairwise comparison of mitochondrial DNA sequences in stable and exponentially growing populations. Genetics, 129, 555-562.

TAïB, z. 1992. Branching Processes and Neutral Evolution. Springer, New York.

TAVARÉ, S. 1984. Line-of-descent and genealogical processes, and their applications in population genetics models. Theor. Pop. Biol., 26, 119-164.

TAVARÉ, s. 1989. The genealogy of the birth, death, and immigration process. In: Feldman, M. (ed.) Mathematical Evolutionary Theory, pp. 41-56. Princeton University Press, Princeton, NJ.

THOMPSON, E. A. 1975. Human Evolutionary Trees. Cambridge University Press, Cambridge.

WOLFRAM RESEARCH 1992. Mathematica, version 2.2. Wolfram Researcy, Inc., Champaign, IL. 